\title{
Realization on the interactive remote video conference system based on multi-Agent
}

\author{
Yan ZHENG ${ }^{1}$, a, Wen Ting LI ${ }^{2}$, Li ZENG $^{3}, \mathrm{Yu} \mathrm{GE}^{1}, \quad$ Xiao Yan CAI ${ }^{1}$, Xian Yu MENG ${ }^{1}$ \\ ${ }^{1}$ Military Information Room, Wuhan Ordnance N.C.O Academy of PLA, Wuhan, 430075 \\ ${ }^{2}$ China Electric Power Research Institute, Wuhan, 430074 \\ ${ }^{3}$ Language Training room, Wuhan Ordnance N.C.O Academy of PLA, Wuhan, 430075
}

\begin{abstract}
To make people at different places participate in the same conference, speak and discuss freely, the interactive remote video conferencing system is designed and realized based on multi-Agent collaboration. FEC (forward error correction) and tree P2P technology are firstly used to build a live conference structure to transfer audio and video data; then the branch conference port can participate to speak and discuss through the application of becoming a interactive focus; the introduction of multi-Agent collaboration technology improve the system robustness. The experiments showed that, under normal network conditions, the system can support 350 branch conference node simultaneously to make live broadcasting. The audio and video quality is smooth. It can carry out large-scale remote video conference.
\end{abstract}

KEY WORDS : multi-Agent; interactive; forward error correction; Peer to Peer; video conference

\section{Introduction}

In daily work, we must participate in many large and small meetings and seminars, but because of a variety of reasons, such as in business trip, in different branch or sick; we cannot attend it in person. The above problem can be well solved by remote video conference system which is developed by computer network and multimedia technology. At the same time, this system can greatly improve the interactivity, through which the remote participants can discuss and communicate with each other through audio and video, letting all participants have face to face experience. In the course of design and implementation the system, the FEC forward error correction coding, P2P technology and multiple Agent theory are adopted, which greatly improved the quality of audio and video services and enhance the robustness, maintainability and adaptability of the system.

\section{Related contents}

\footnotetext{
${ }^{\mathrm{a}}$ Corresponding author: zy397037172@163.com
}

\section{FEC forward error correction}

FEC error correction was used in the correction of bit stream. In this system, it is used to make group data error correction. It encodes the original data firstly to produce a certain amount of redundant data, and then the sender sends out the $\mathrm{N}$ groups. As long as we can receive any $\mathrm{K}$ groups of the $\mathrm{N}$ groups at the receiving end, the original data can be returned, which greatly reduce the probability of flowers screen caused by echo video when there is bad network packet loss.

\section{Multi-Agent technology}

Intelligent Agent, a computer program running in mobile environment, has highly autonomous ability, which can accept entrust of another entity like user, system or machine, and provide aid and service for it. Under the drive of the target it is also capable of initially carrying out the action including social contact and study, to sense and adopt the change of mobile environment, and cope it with properly response. As Agent has the autonomous ability, it itself can use its decision-making mechanism to select the strategy and make response without users' monitor and guidance. The sociality of 
Agent realizes the function that one Agent can communicate with another Agent, which can offer better service for users.

\section{P2P Tree}

A Multicast distribution tree is constructed in accordance to the conference situation, which is Audio and video dispatching strategy with push-pull combination based on tree structure. The branch conference node can link child node, distributing audio video data downward and setting the allowed maximum layers of tree by preset to make sure the postpone time of bottom node will not too big $^{[1]}$.

\section{System functional structures}

The structure diagram of the interactive remote video conferencing system based on multi-Agent is as follow (Fig.1).

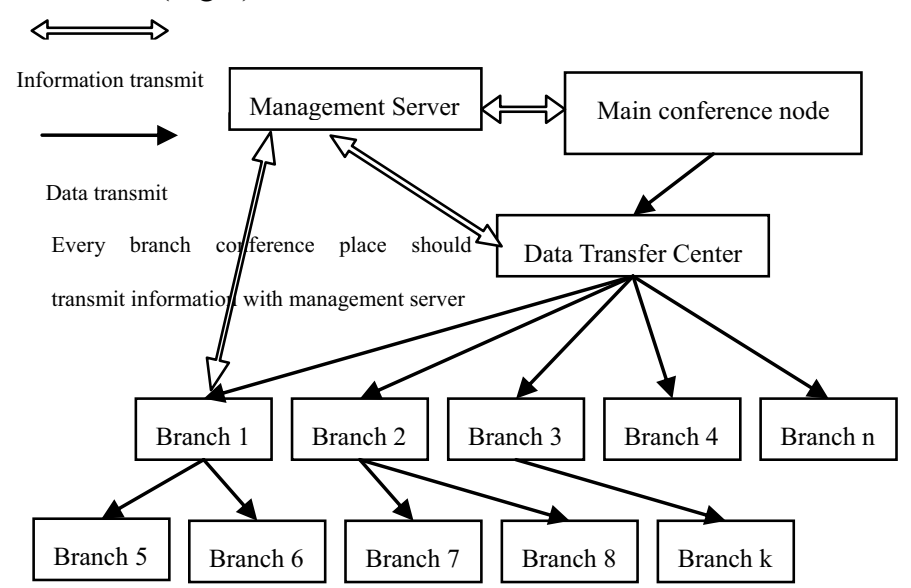

Fig.1 The structure diagram of the interactive remote video conferencing system based on multi-Agent

The system was divided into four modules: management Server, DTC (Data Transfer Center), main conference node, branch conference node.

The scene of conference supports a main conference node and many branch conference nodes. After having applied interactive node, people can communicate, talk and discuss in branch conference place. You can join in the corresponding conference place, if you input Server IP, conference ID and local conference place' name and password in branch conference place port. The system can support more than one live branch conference branches at one time. The branch conference place which has spare bandwidth can be used as father node to send audio and video data to its child node of branch conference place port.

At the same time, the system has interactive function. Branch conference place can be applied as conference place interactive point, from which speech can be discussed and local audio and video data can be send out, and all conference place ports connected with this conference place port can receive the audio and video data. It guarantees that all branch conference places have opportunity to take part in the communication.

\section{System realization}

\section{Interactive focus}

Branch conference place can be applied as conference place interactive focus. After having approved by main conference place, it transmits the local audio and video data to DTC, and DTC transmits the data to main conference place and each branch conference place. In this way all receive port in the conference place can watch and listen the speech and discussion of the branch conference place. The system can support no more than five interactive focuses to take part in conversation and discussion simultaneously.

Applying branch conference place to be an interactive focus is realized as following (Table. 1).

Table. 1 branch conference node applying focus

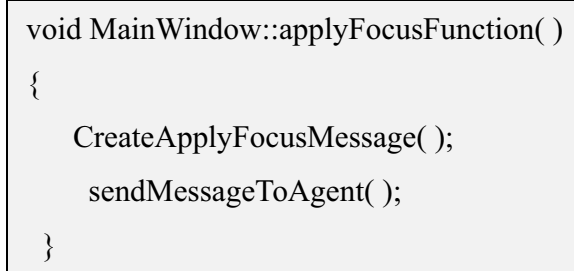

Interactive focus transmitting local audio and video data to DTC is realized as following (Table. 2).

\section{The multi-Agent}

Information processing Agent, data processing Agent and new node processing Agent were set in the system. They coordinate with each other to finish the organization and control of the system. ${ }^{[3]}$

Every branch conference port requires sending information to management Server. As there are different 
kinds of information, branch conference place port needs to engage in corresponding operation according to different information. In order to promote system processing capacity, an information processing Agent was set.

Table. 2 Interactive focus transmitting local audio and video

\section{data to DTC}

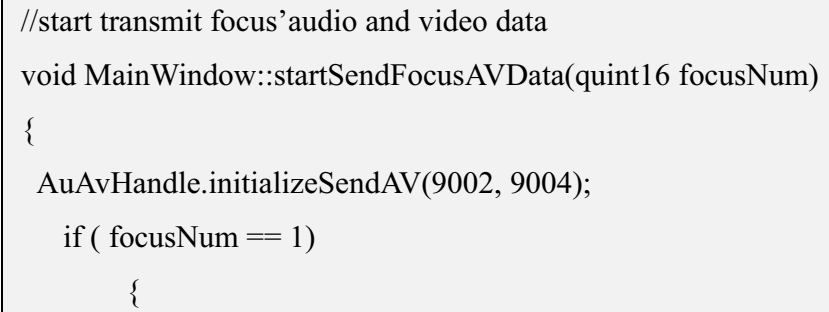

DTC will transmit a great deal of data to branch conference places. Since the data have big volume, branch conference port can hardly process all of them in time. If the data processing Agent is set, it can receive data and decode it into audio and video format, which will be transmitted to matched port and played.

As to those new nodes which will add to the system, emphasis should place on considering that under which node it connect to receive data in high quality. The new added node Agent can finish this function.

The simple reaction Agent is used in the system. Its function procedure is as follow.

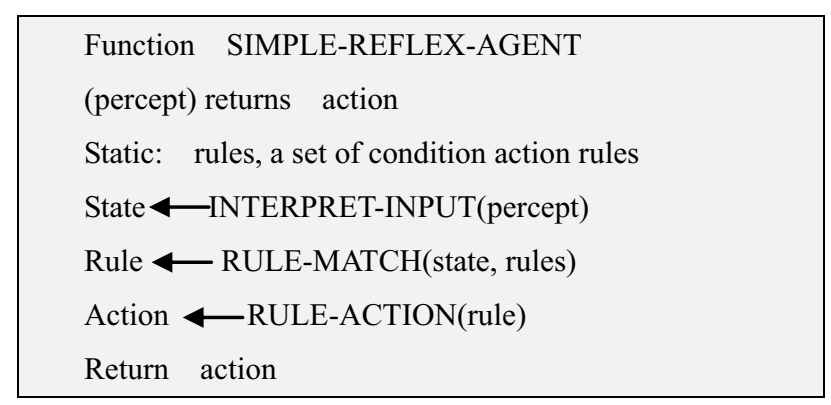

The simple reaction Agent is relatively simple, which should sense the current input state, and based on it, find matched rule of the state in predefined state set. The corresponding operation is finished according to this rule. Multi-Agent can work together to finish complicate task.

\section{Key Technology}

\section{Forward error correction coding}

By using the technology of FEC (forward error correction coding), receive port can use the received normal data package and redundancy data packet to decode the original audio and video data even though there are a few packet loss ${ }^{[5]}$.

The technology of forward error correction coding is dramatically decrease the influence which is caused by network packet dropout for the quality of audio and video in system. When the rate of packet lost less than $20 \%$, FEC can decode and repair it into normal audio and video to avoid flower screen.

\section{Tree P2P}

The Management Server is responsible for constructing, maintaining and upgrading multicast tree, which stores information of every node and supports node to find father node. The system will be more robust when some node leave or some node is failure. Typically, father node push data to their son nodes actively according to their data in NICE system ${ }^{[4]}$.

After having acquired part of data DTC by using pull method as startup (pull), it transmits data package to its child node without requiring that the child node asks for data package (push). Pull model is of good robustness, which can work properly in highly disturbed P2P environment. Push model can effectively reduce cumulative delay observed at each node. Combing pull 
with push model can deal with dynamic question of child node efficiently without producing high startup delay.

P2P technology can make good use of spare upstream broadband of every branch conference places' terminal to transmit video and audio data for new added node of branch conference place, which can partake DTC load volumes to increase conference places and guarantee the smoothness of audio and video.

\section{Experiment and Results}

The system had tested in computer lab, and the result showed that the testament is successful.

The system increased delay time when conference places increased (the lever number of P2P Multicast tree is within predicative value).It is shown in Fig. 2. Under normal network condition, delay time was controlled within $8 \mathrm{~s}$ when branch conference place increased. The test result showed that the delay time was different under different father node.

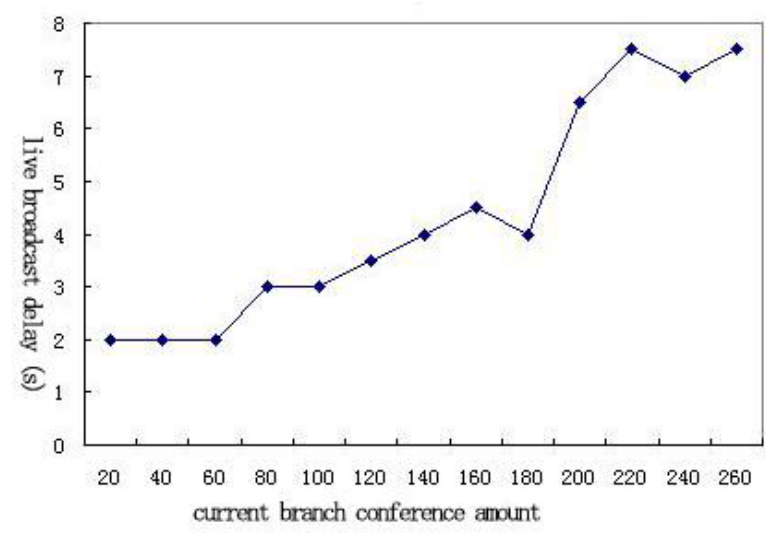

Fig.2 The relation between live broadcast delay and branch conference amount

When the current branch conference places added, the change of dropout rate is shown in Fig.3. Since FEC forward error correction used in the system could make code, no blurred screen was produced when the dropout rate was less than $20 \%$. However, when the number of conference places were approaching to the limit that network card of Server can undertake, the dropout rate would be serious, and part of conference places could produce blurred screen.

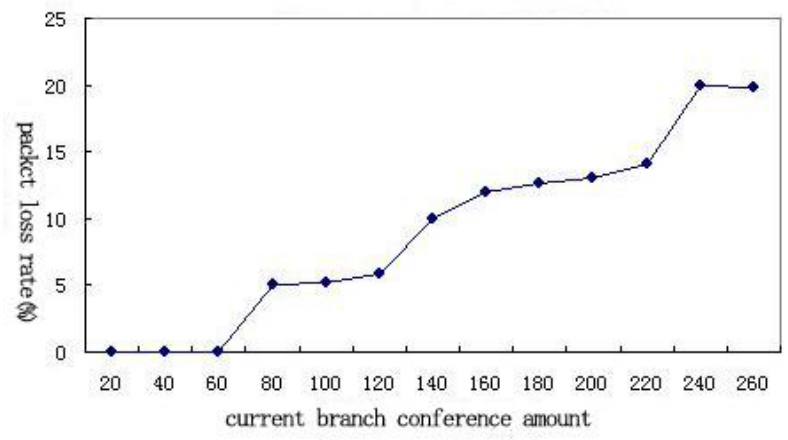

Fig.3 The relation between packet loss rate and branch conference amount

When the branch conference place increased, the change of the Server bandwidth occupancy rate is shown in Fig. 4. Since DTC server used kilomega broadband, it supported support 350 nodes simultaneously to make live broadcasting.

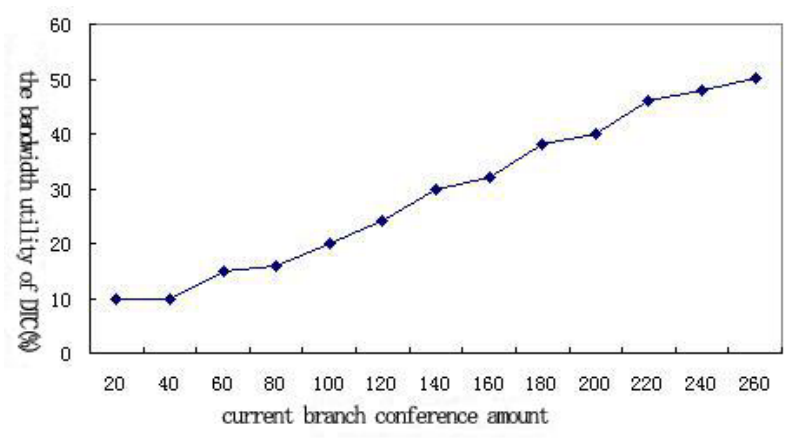

Fig.4 The relation between the bandwidth utility of DTC and branch conference amount

\section{Conclusion}

At present, the remote video and audio conference system is equipped in our academy to apply and test. The function and stability of this system has been widely recognized after having been applying it in several large-scale video and audio conferences. The imported Multi-Agent cooperation theory technology enhances the robustness and stability of the system dramatically. Meanwhile, FEC forward error correction coding and $\mathrm{P} 2 \mathrm{P}$ tree are used to make sure that the system can transmit video and audio with good quality and liquidity. The applicability and maintainability of the system has been improved. 


\section{References}

[1] Segarra J, Cholvi V. Convergence of periodic broadcasting and video-on-demand[J]. Computer Communications, 2007, 30 (5): $1136-1141$.

[2] Cheng B, Stein L, Jin H, et al. GridCast: Improving peer sharing for P2P VoD[J]. ACM Transactions on Multimedia Computing, Communications, and Applications (TOMCCAP), 2008, 4 (4): 1-31.

[3] Cheng Feng, Study of Agent Reliability under Multi-Agent Cooperative Decision-Making[J]. Pattern Recognition And Artificial Intelligence;2008.10. Vol.21, No.5

[4] Zhong Yuzhuo et al. Media Streaming and Video Server[M]Beijing: Tsinghua University Press, 2003.

[5] Wu Zhigang, Bai Guangwei, Adaptive FEC based diffserv model for real-time media streaming[J]. Computer Engineering And Design;2009,30(6) 